\title{
A Half Mode Inkjet Printed Tunable Ferrite Isolator
}

\begin{abstract}
A novel half mode waveguide based ferrite isolator design is presented in this work. For the first time, tunability of the isolation band is demonstrated for a ferrite isolator. Instead of using the conventional antisymmetric bias the isolator requires a single direction of the magnetic bias field due to the half mode operation. Yttrium Iron Garnet (YIG) is used as the substrate for the device. The metallic walls of the waveguide are realized using inkjet printing. The magnetic biasing applied to the waveguide causes the RF waves to experience negative permeability in one direction of propagation hence providing isolation for this direction. For an applied bias of $\mathbf{3 0 0 0}$ Oe, the device provides a maximum isolation figure of merit of $76.7 \mathrm{~dB}$ at $7.5 \mathrm{GHz}$. The isolation band can be controlled by changing the applied magnetostatic bias. As the bias is varied from 1500 Oe to 3500 Oe the center frequency of the isolation band varies from $4.45 \mathrm{GHz}$ to $9 \mathrm{GHz}$. The measured response of the isolator shows that it can be integrated in any RF system requiring lower cost and good isolation.
\end{abstract}

Index Terms - isolator, ferrite, waveguide, isolator figure of merit (IFM)

\section{INTRODUCTION}

Microwave isolators are commonly used in the RF transmitters between the power amplifier and the antenna to avoid any unwanted signals reaching the amplifier. The presence of these spurious signals can cause intermodulation distortion hence reducing the overall efficiency of the transmitter chain [1]. Typically, the realization of these isolators requires non-reciprocal mediums such as ferrites. Various isolator designs based on bulk ferrite materials have been reported in literature. These designs operate on the principle of resonance absorption (negative permeability) [2], Faraday rotation [3] and field absorption [4]. Most of these designs are based on waveguides due to their superior RF performance in terms of loss and power handling capability.

Generally waveguide based ferrite isolators require antisymmetric bias which means that the magnetostatic bias in one of half of the waveguide is opposite to the bias in the other half (along the width of the waveguide) as shown in Fig. 1(a) [5]. Such a design needs two different magnetic sources to provide the required directions inside the medium. As opposed to this, if a half mode waveguide is used for the wave propagation then a single magnetic source can be used for generating the isolation band, Fig. 1(b). Previously, half mode ferrite based substrate integrated waveguide (SIW) has been used for phase shifter design [6]. In that work it is shown that a half mode waveguide can improve the efficiency by reducing the number of magnetostatic bias sources. The same concept can be used for an isolator design and will be investigated in this paper.

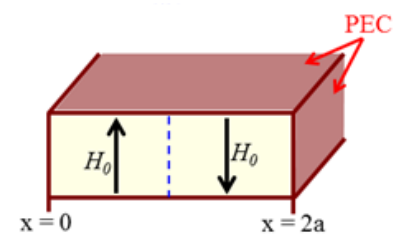

(a)

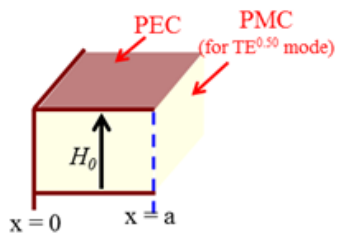

(b)
Fig.1. Ferrite filled waveguide based isolator designs (a) Full mode anti-symmetrically biased (b) Half mode with a single direction of bias

To realize the half mode isolator design on a magnetic substrate, inkjet printing is used for the device fabrication. Inkjet printing has emerged as one of the most efficient and low cost fabrication techniques in recent years. Its additive nature allows the reduction in material wastage as compared to the traditional photolithography based fabrication processes. Furthermore, it does not require any mask which reduces the cost of the device manifolds. Finally it is very suitable for mass production because of its roll-to-roll printing ability. Several RF devices such as inductors, capacitors, antennas etc have been reported which rely on inkjet printing for their fabrication [7], [8].

In this paper, inkjet printing is used to realize a half mode isolator design using YIG substrate. For the first time, a half mode isolator design is reported using any magnetic substrate. Furthermore, the tunability of the isolation band is studied by varying the applied magnetostatic field across the device. The isolation center frequency is varied from $4.45 \mathrm{GHz}$ to $9 \mathrm{GHz}$ by changing the applied bias from 1500 Oe to 3500 Oe. A maximum isolation figure of merit (IFM) of $76.7 \mathrm{~dB}$ is measured from the device.

\section{THEORY AND DESIGN}

Ferrites are known to be anisotropic materials due to their permeability. When a ferrite medium is biased with a normal magnetostatic field, it generates a permeability given be,

$$
\mu_{e f f}=\frac{\mu^{2}-\kappa^{2}}{\mu}
$$

where $\mu$ and $\kappa$ are the elements of the permeability tensor and are given be Polder's equation when the ferrite is completely saturarted [1]. Above equation can be expressed in terms of poles and zeros as,

$$
\mu_{e f f}=\mu_{0} \frac{f^{2}-f_{z}^{2}}{f^{2}-f_{p}^{2}}
$$


In the above equation $f_{\mathrm{p}}$ and $f_{\mathrm{z}}$ are the poles and zeros of the function while $\mu_{0}$ is the free space permeability. The zero is located at $f_{z}=f_{0}+f_{m}$ while the pole is located at $f_{p}=\sqrt{f_{0}\left(f_{0}+f_{m}\right)}$, where $f_{0}=\mu_{0} \gamma H_{0}$ is the gyromagnetic resonant frequency, $f_{m}=\mu_{0} \gamma M_{s}$ is the magnetization frequency, $\gamma$ is the gyromagnetic ratio constant, $H_{0}$ is the internal magnetostatic bias field, and $M_{s}$ is the saturation magnetization. A plot of permeability against the frequency for a saturated ferrite is shown in Fig. 2. The plot shows that $\mu_{\text {eff }}$ has negative values in the range $f_{p}<f<f_{z}$. So if a wave is propagating inside the waveguide within this band, it will be absorbed by the material resulting in high insertion loss. As a result, the waveguide can be used as an isolator within this range of frequencies. Furthermore, since $f_{p}$ and $f_{z}$ are functions of $H_{0}$ and $M_{s}$, it is possible to control the isolation band's centre frequency. If for a same material the applied bias is varied across it then this negative permeability band can be tuned to control the isolation band. In this way a tunable isolator can be realized.

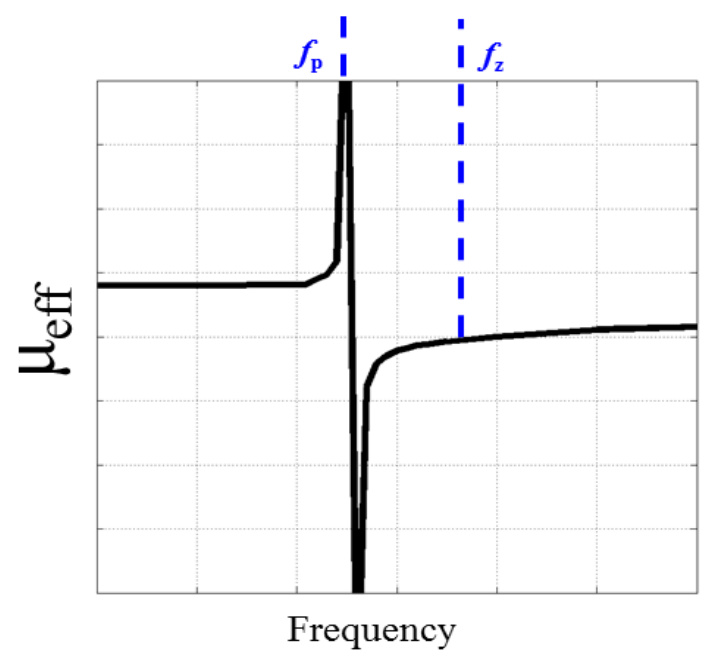

Fig. 2. Permeability of ferrite substrate versus frequency

To verify the theory, a half mode isolator design is used in this work as shown in Fig. 3. The isolator is designed on a YIG1780 substrate with a saturation magnetization, $4 \pi M_{s}$ of $1780 \mathrm{G}$. This value results in a magnetization frequency of 5 GHz. Generally the lower limit for the operation frequency of a ferrite based design is restricted to its magnetization frequency. However, since the design in this work is operated in the saturated state, this lower limit will not be imposed on the device. Therefore, the waveguide will be studied starting from its cut off frequency which is around $3.9 \mathrm{GHz}$. The waveguide is fed with a $50 \Omega$ microstrip line which is connected to the waveguide edge using a tapered microstrip line. The dimensions of the waveguide are optimized using Ansys HFSS and are shown in Fig. 2.

\section{FABRICATION AND MEASUREMENTS}

Dimatix 2831 materials printer is used for the printing of metallic layers on the YIG substrate. Silver-organo-complex
(SOC) based ink is used for the printing of the top and bottom conductor layers of the waveguide. The conductivity of the printed metal can be increased by printing multiple layers of ink. Six layers of ink are printed to realize the isolator. All the walls of the waveguide are printed using the SOC ink including the vertical wall. After printing, the substrate is heated up to $80^{\circ} \mathrm{C}$ (using Infrared heating) for $5 \mathrm{~min}$. The size of the isolator is $4.8 \mathrm{~mm} \times 24 \mathrm{~mm} \times 0.4 \mathrm{~mm}$. The final fabricated image of the isolator is shown in Fig. 4.

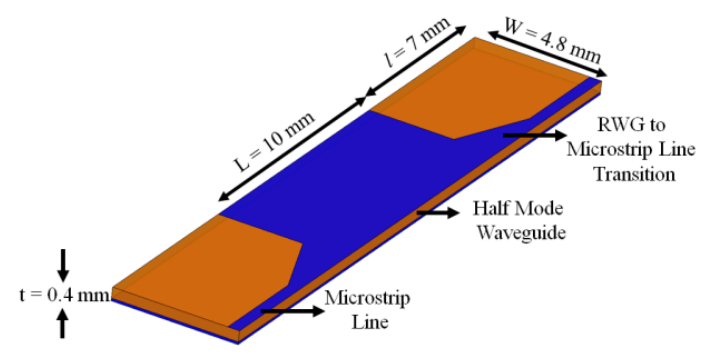

Fig. 3. Half mode isolator design

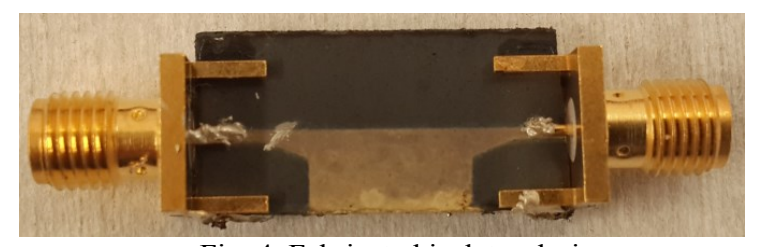

Fig. 4. Fabricated isolator design

For the measurements of the isolator in the magnetized state it is placed in between the poles of an electromagnet. The bias strength of the electromagnet is varied from $0 \mathrm{G}$ to $3500 \mathrm{Oe}$. At $1500 \mathrm{G}$, the isolation starts to appear in the device. The $\mathrm{S}$ parameters of this measurement are shown in Fig. 5. The isolator exhibits an insertion loss of $3.5 \mathrm{~dB}$ (including the transition) with an isolation of $58.2 \mathrm{~dB}$. The zero frequency of the isolation band is below the cut off frequency of the waveguide. This can be deduced from Fig. 5 where the isolation of the waveguide is high below $3.9 \mathrm{GHz}$ but the transmission is also getting attenuated. For example at 3.8 $\mathrm{GHz}$, the isolation is almost $30 \mathrm{~dB}$ with a transmission of 10 $\mathrm{dB}$. Therefore, it is difficult to exactly locate the zero frequency for this bias. In any case a good isolation is measured from the device for 1500 Oe strength of magnetic fields. The measured isolation figure of merit (IFM = Isolation-Transmission) is shown in Fig. 6. The maximum value of IFM is measured to be $54.6 \mathrm{~dB}$. This value is quite comparable to the values that are reported in the literature [2][5].

The biased measurements of the waveguide show good isolation performance at 1500 Oe. Now the applied magnetostatic field is varied across the device to tune the isolation band. The bias fields are varied from 1500 Oe to 3500 Oe. At the same time the pole $\left(f_{\mathrm{p}}\right)$ and zero $\left(f_{\mathrm{z}}\right)$ frequencies of the device are recorded. The tuning of $f_{\mathrm{p}}$ and $f_{\mathrm{z}}$ versus the applied magnetostatic bias is shown in Fig. 7. 


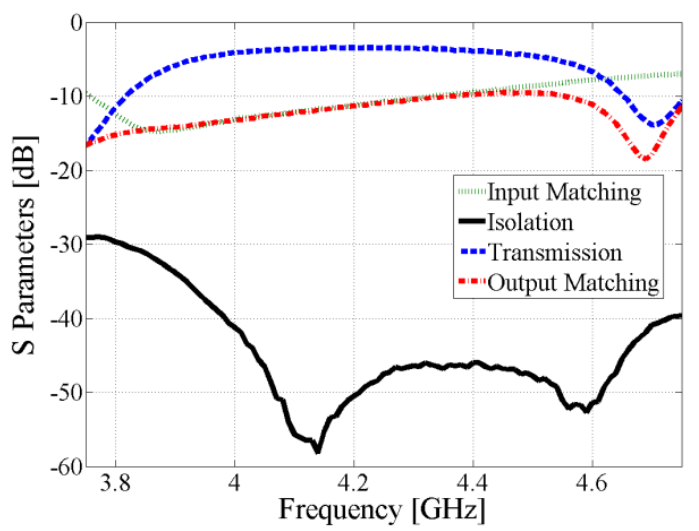

Fig. 5. Measured S parameters of the isolator for a bias of $1500 \mathrm{Oe}$

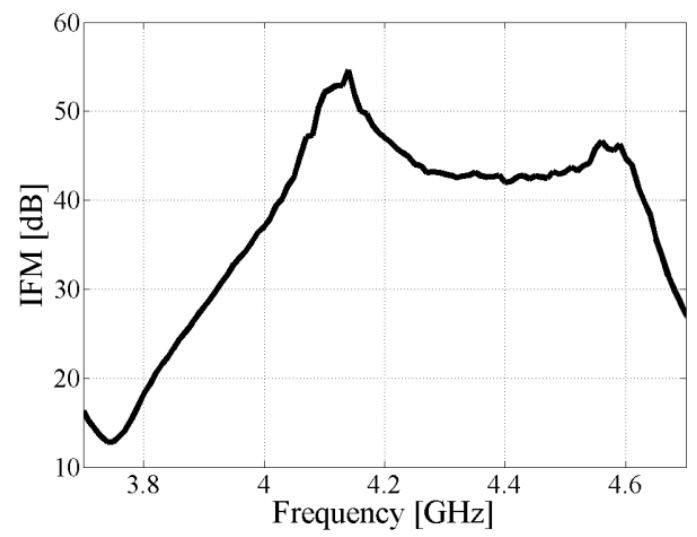

Fig. 6. Measured IFM of the isolator for a bias of $1500 \mathrm{Oe}$

Assuming the two frequencies to be the egde of the isolation band, the center frequency of the isolator is calculated and is observed to be varying from $4.45 \mathrm{GHz}$ to $9 \mathrm{GHz}$. The variation of IFM with the applied bias is shown in Fig. 8. The IFM values generally remains above $50 \mathrm{~dB}$ with the maximum value of $76.7 \mathrm{~dB}$ observed at $7.5 \mathrm{GHz}$ for a bias field strength of 3000 Oe. Increasing the bias beyond 3500 Oe can further tune the isolation band but with a higher insertion loss due to poor input and output matching. These measurements provide a proof-of-concept that by varying the magnetic field across the isolator the band of isolation can be varied. To the best of author's knowledge this is the first time that a half mode isolator design has been studied using ferrite material for tunable applications.

\section{CONCLUSION}

A ferrite filled half mode tunable isolator design is reported in this work. It is for the first time that a half mode inkjet printed isolator is reported using a ferrite medium that demonstrates frequency tuning. The isolator shows a minimum insertion loss of $3.5 \mathrm{~dB}$ with a maximum IFM of $76.7 \mathrm{~dB}$. The center frequency of the isolator is varied from $4.45 \mathrm{GHz}$ to $9 \mathrm{GHz}$ by changing the applied bias from 1500 Oe to 3500 Oe. This design shows that using a low cost fabrication technique (inkjet printing) an efficient and tunable microwave device can be realized for high end applications.

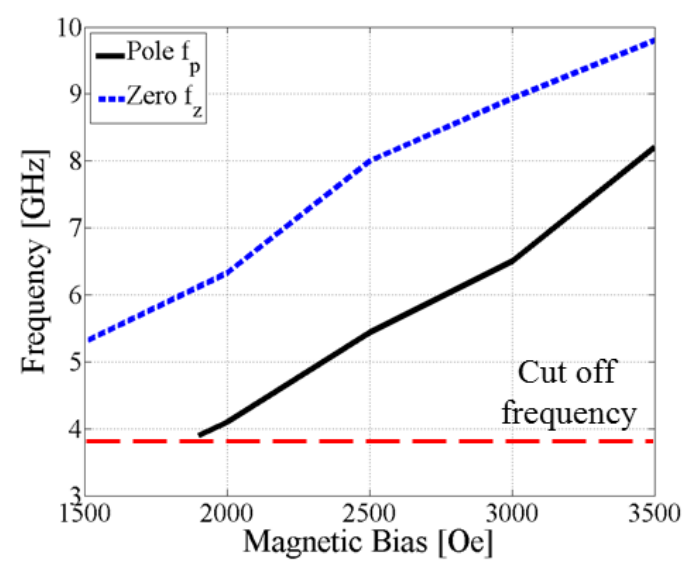

Fig. 7. Tuning of isolation bandwidth of the half mode isolator design

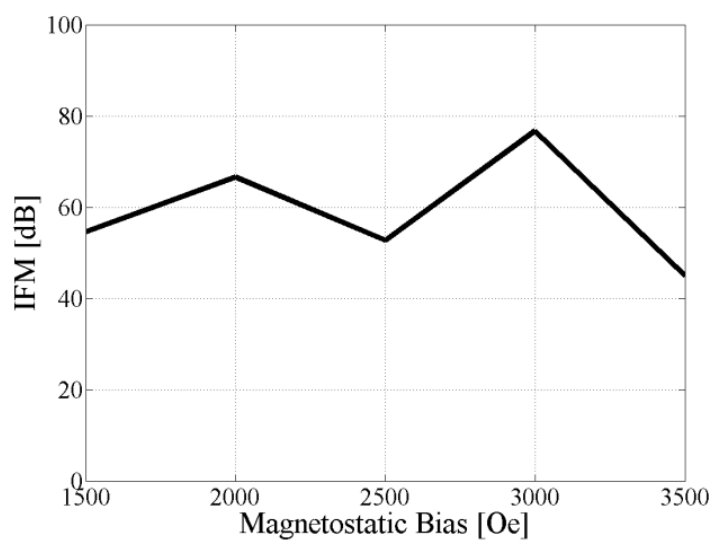

Fig. 8. Variation of IFM with the applied bias

\section{REFERENCES}

[1] D. M. Pozar, "Microwave Engineering", $3^{\text {rd }}$ Edition, chapter 9, pp. 465, 2005.

[2] M. F. Farooqui, et. al., "Inkjet printed ferrite-filled rectangular waveguide X-band isolator," IEEE MTT-S International Microwave Symposium, pp. 1-4, 2014.

[3] F. Fesharaki, et. al., "Broadband substrate integrated waveguide edge-guided mode isolator," Electronics Letters, vol.49, no.4, pp.269-271, 2013

[4] D. Deslandes, et. al., "Accurate modeling, wave mechanisms and design considerations of a substrate integrated waveguide," IEEE Transactions on Microwave Theory and Techniques, vo1.54, no.6, pp.2516-2526, June 2006.

[5] C. K. Seewald, et. al., "Ferrite-filled antisymmetrically biased rectangular waveguide isolator using magnetostatic surface wave modes," IEEE Transactions on Microwave Theory and Techniques, vol. 58, pp. 1493-1501, June 2010.

[6] F. A. Ghaffar, et. al., "A Partially Magnetized Ferrite LTCC Based SIW Phase Shifter for Phased Array Applications", IEEE Transactions on Magnetics, vol.51, no.6, pp.1-8, 2015.

[7] S. Molesa, et. al., "High quality inkjet-printed multilevel interconnects and inductive components on plastic for ultra-low cost RFID applications," Proceedings of Material Research Society, vol. 769, pp. 8:3-1, 2003.

[8] T. Zhang, et. al., "A Novel Multiband Planar Antenna for GSM/UMTS/LTE/Zigbee/RFID Mobile Devices," in IEEE Transactions on Antennas and Propagation, vol. 59, no. 11, pp. 4209-4214, Nov. 2011. 